\title{
EchoGéo
}

45 | 2018

Déclinaisons géographiques du changement social en Iran

\section{Déclinaisons géographiques du changement social} en Iran

Introduction

Amin Moghadam, Mina Saïdi-Sharouz et Serge Weber

\section{(2) OpenEdition}

Journals

Édition électronique

URL : https://journals.openedition.org/echogeo/15962

DOI : $10.4000 /$ echogeo. 15962

ISSN : 1963-1197

Éditeur

Pôle de recherche pour l'organisation et la diffusion de l'information géographique (CNRS UMR 8586)

Référence électronique

Amin Moghadam, Mina Saïdi-Sharouz et Serge Weber, « Déclinaisons géographiques du changement social en Iran », EchoGéo [En ligne], 45 | 2018, mis en ligne le 05 novembre 2018, consulté le 11 août 2021. URL : http://journals.openedition.org/echogeo/15962 ; DOI : https://doi.org/10.4000/echogeo. 15962

Ce document a été généré automatiquement le 11 août 2021.

EchoGéo est mis à disposition selon les termes de la licence Creative Commons Attribution - Pas d'Utilisation Commerciale - Pas de Modification 4.0 International (CC BY-NC-ND) 


\title{
Déclinaisons géographiques $d u$ changement social en Iran
}

\author{
Introduction
}

Amin Moghadam, Mina Saïdi-Sharouz et Serge Weber

1 Proposer un numéro thématique en géographie sur un pays comme l'Iran dans la deuxième moitié des années 2010 est à la fois une entreprise passionnante et un défi. Depuis quelques années, la République islamique d'Iran attire tous les regards. L'élection de $\mathrm{H}$. Rohani à la présidence en 2013 a permis le retour des modérés au pouvoir après les deux mandats du conservateur $M$. Ahmadinejad. La réouverture des négociations multilatérales autour du fameux « dossier nucléaire » iranien, qu'on a pu interpréter comme une sorte de dégel des relations entre États-Unis et Iran, a abouti aux accords dits de Genève en 2015, qui programmaient la levée progressive des sanctions économiques et diplomatiques internationales à l'égard du pays.

\section{Les sanctions internationales, un paramètre incontournable du baromètre social iranien}

2 Ces sanctions sont évidemment un facteur très prégnant pour qui s'interroge sur la société iranienne et sur la géographie humaine du pays. Initiées à la suite de la révolution de 1979, les sanctions internationales ont successivement connu des périodes d'allègement et de renforcement, mais leur présence constante, surtout pendant les années 1980 et 2010 où elles ont été plus fortes, joue en continuation sur tous les aspects de la vie économique, sociale et quotidienne (Coville, 2000). Leur renforcement considérable au milieu des années 2000 a eu pour conséquence un isolement plus fort du pays, qui s'est accompagné du départ de nombre d'entreprises occidentales qui contribuaient à l'activité de secteurs entiers, notamment industriels, mais aussi de la chute du commerce international, à l'import ou à l'export. Étant mise à l'écart du SWIFT (Society for Worldwide Interbank Financial Telecommunication, ou système interbancaire permettant les règlements internationaux), la République islamique s'est trouvée dans une situation difficile pour le règlement des transactions internationales 
et pour sa politique monétaire. Les sanctions économiques internationales ont participé au creusement des inégalités : même si le taux de pauvreté n'a cessé de baisser depuis le début des années 1980, les écarts de revenus entre les classes se sont accentués (Salehi-Isfahani, 2017).

Les sanctions diplomatiques ont de leur côté fortement limité, pour les Iraniens, la possibilité de circuler hors d'Iran, les politiques d'attribution de visas ayant été durcies par de nombreux pays, alors même que les échanges ont encore plus dépendu des conditions d'organisation des voyages à l'étranger (Moghadam et Weber, 2015; Mohabbat-Kar, 2015). En effet, la dimension transnationale est incontournable pour comprendre l'Iran contemporain et rappelle que les mobilités internationales, depuis et vers l'Iran - notamment ce qu'on pourrait appeler la «diaspora » iranienne, même si ses contours sont extrêmement flous et kaléidoscopiques - ont une place importante dans la société iranienne. Une partie non négligeable des personnes qui mènent leurs recherches sur l'Iran ont fait au moins un séjour d'études à l'étranger, vu la part des Iraniens dans les mobilités internationales étudiantes et de jeunes diplômés (Naficy, 1993 ; Colby, Friedlander et Kelley, 1993 ; Adelkhah, 2001 ; Moghadam, 2016).

De même que face à toute situation d'embargo, des échanges alternatifs ont été maintenus ou développés, par exemple avec des pays qui ne se sont pas engagés aussi clairement aux côtés des États-Unis et des autres États imposant ces sanctions, au moyen de reconfigurations des circulations et des flux vers d'autres destinations, ou encore par le biais de circulations informelles. Ces modalités alternatives d'ouverture au reste du monde s'observent à la fois par le haut et par le bas, tant dans les secteurs formel qu'informel, et impliquent des pays aussi divers que la Chine et la Malaisie ou encore les pays frontaliers comme la Turquie ou les Émirats arabes unis.

5 L'annonce de la levée programmée des sanctions, après 2016, a vivement intéressé les milieux d'affaires et de coopération de nombreux pays. Une vague de publications, tant scientifiques que de vulgarisation, a vu le jour, dressant un portrait de l'actualité de l'Iran, faisant notamment état des transformations récentes qu'ont connues les sphères sociale, économique et politique de cette puissance régionale située entre MoyenOrient et Asie centrale (Djalili et Kellner, 2016). Cette perspective ouvrait des potentialités de développement comme les investissements directs étrangers ou le tourisme. De fait, le tourisme international a repris de sa vigueur et beaucoup d'entreprises qui avaient quitté le pays ont commencé à opérer un retour et à réactiver les joint-ventures que le renforcement des sanctions avait interrompus. Pour autant, le raidissement ultérieur de l'administration américaine vis-à-vis de l'Iran depuis l'élection de D. Trump en 2017 a freiné le processus de levée des différentes sanctions sectorielles, dans la mesure où dès mai 2018, les États-Unis ont remis en cause leur engagement. La population iranienne attend donc encore et souffre de l'asphyxie économique qu'entraîne cette volte-face. La réactivation, plus caricaturale encore, du répertoire argumentaire manichéen de G. W. Bush par D. Trump, a contraint les autorités iraniennes et une partie de l'opinion publique à promouvoir des discours de plus en plus méfiants vis-à-vis des États-Unis et de leurs partenaires et à renouveler un discours nationaliste qui s'était atténué. Cela n'ôte rien au fait que, pour la majorité des habitants, l'ouverture au reste du monde, la possibilité de circuler et de relancer l'import-export restent une nécessité difficile à mettre en œuvre mais primordiale.

6 L'appel à article de ce numéro émanait de ce pari, fait en 2015-2016, que la levée programmée des sanctions allait s'accompagner d'une reprise des recherches en 
sciences sociales sur les nouveaux visages de la société iranienne, l'émergence d'activités économiques nouvelles (tourisme, commerce, entreprenariat), l'émergence de nouveaux types d'acteurs et d'idées dans les transformations spatiales à l'œuvre, tant dans les espaces urbains qu'à la campagne. De nombreux changements ont été amorcés depuis plus d'une décennie ou même davantage dans la société et l'économie iraniennes (Coville, 2007), qui remettent en question les nombreuses idées reçues qui circulent sur ce pays (Adelkhah, 2013). L'hypothèse était alors partagée que la levée des sanctions aurait eu des effets multiples, à toutes les échelles, sur les recompositions spatiales liées à une ouverture attendue, que ce soit dans le domaine du foncier, de la production du bâti, des grands projets urbains et des infrastructures, sans oublier les activités commerciales ou touristiques. Mais force a été de constater, depuis lors, que la levée effective des sanctions se fait attendre, et que les difficultés restent présentes.

\section{Quelles géographies de l'Iran ? Entre area studies et renouvellements transdisciplinaires}

7 Ce projet de dossier thématique envisageait également de faire la part belle à la dimension géographique des études sur l'Iran contemporain. En effet, la géographie est d'une certaine manière le parent pauvre des diverses traditions académiques représentées dans ce vaste champ interdisciplinaire des area studies qui se reconnaissent sous la bannière des Iranian studies, animé par des congrès scientifiques, des associations scientifiques, des collections éditoriales et des revues spécialisées comme Studia iranica ou Iranian Studies (Hourcade et Richard, 1993). Dans ce champ, très internationalisé (qu'on retrouve aussi bien en Iran que dans le monde académique anglophone, francophone et germanophone principalement) et fortement arrimé aux études de langue et de civilisation persane, certaines disciplines sont bien représentées, comme l'histoire et la géopolitique historique (Digard, Hourcade et Richard, 2007 [1996]), l'anthropologie (Digard, 1981; 1988; Bromberger, 1998), l'anthropologie politique, notamment du chiisme (Richard, 1980; Mervin, 2007), l'économie (Coville, 2000), sans oublier l'archéologie (Mousavi et Naseri-Moghaddam, 2009), l'histoire de l'art ou encore la linguistique.

Un champ interdisciplinaire comme les relations internationales a évidemment eu une certaine prédilection pour un pays situé au cœur de ce qu'on appelait l'échiquier moyen-oriental ou le «Grand jeu » au début du XX $\mathrm{XX}^{\mathrm{e}}$ siècle, qui n'a cessé de se rejouer, en particulier autour de l'enjeu des hydrocarbures (Djalili, 2005 ; Giblin, 2018). De fait, ce pays a connu une révolution qui a eu des effets de taille sur l'histoire mondiale. Cette révolution reste l'une des rares à avoir instauré une situation post-révolutionnaire effectivement durable (près de quatre décennies) et à avoir mis en place des politiques sociales indépendamment de la dimension théocratique (Harris, 2017). Sans oublier que le pays a connu une guerre qui reste la plus longue du XXe siècle. À cela on ajoutera que l'Iran voit aujourd'hui se nouer et de dénouer des alliances potentielles, nouvelles ou réactivées, dans un monde de plus en plus multipolaire. L'Iran tente de s'affirmer comme une puissance régionale, face à d'autres États qui lui font concurrence (comme la Turquie ou l'Arabie saoudite), et face aux grandes puissances comme les Etats-Unis et la Russie, qui contribuent en ce début de siècle à réactiver le "grand jeu " moyenoriental ${ }^{1}$. 
9 Finalement, peu de géographes ont effectué leurs recherches sur l'Iran, et les monographies de géographie générale ou régionale du pays sont rares (Kiyâni Haftlang, 2003). La géographie physique et plus généralement la géophysique ont amplement documenté la question des risques naturels, au premier rang desquels figurent les risques sismiques. En France, Bernard Hourcade a produit de nombreuses analyses inédites, en particulier sur la croissance urbaine et on lui doit une précieuse activité de cartographie qu'on retrouve dans deux atlas qui font toujours autorité, l'un à l'échelle du pays, l'autre centré sur la capitale, Téhéran (Hourcade, 1998; 2003; Habibi et Hourcade, 2005).

Si on considère que la géographie est, comme nombre d'autres disciplines des sciences sociales depuis la diffusion de la critique postmoderne, de plus en plus souvent animée par des champs transdisciplinaires, alors on doit prendre en considération le regain récent de ce qui relève des migration studies et des urban studies associant géographie, anthropologie urbaine, cultural studies et économie politique (Moghaddari, 2015; Moghadam, 2013 ; Malek, 2015).

11 Ces renouvellements récents des études urbaines et des études migratoires, dans des géométries institutionnelles variées et des milieux de recherches plus ou moins académiques, plus ou moins liés aux chercheurs indépendants ou aux artistes, voient les travaux sur l'Iran intégrer les apports de ces renouvellements thématiques (nouveaux objets d'études) ou théoriques (nouvelles grilles d'interprétation). Les entretiens proposés dans les autres rubriques de ce numéro tentent de donner un aperçu de la richesse des réflexions interdisciplinaires sur la société iranienne et la production urbaine, n'émanant pas nécessairement du monde académique. Concernant l'articulation entre anthropologie et économie politique, des travaux comme ceux de Fariba Adelkhah (2012) à partir des voyages des Iraniens à l'étranger ont considérablement renouvelé l'anthropologie de la mobilité et approfondi l'interprétation de l'idée de nation à partir du cas iranien. Ces travaux s'inscrivent dans un mouvement plus large dans les sciences sociales, dépassant le nationalisme méthodologique et mettant l'accent sur la dimension transnationale de la formation de l'identité nationale et territoriale (Kashani-Sabet, 1999; Keddie et Matthee, 2002; Amanat et Veidani, 2012 ; Stone, 2014 ; Chehabi, Jafari et Jofroudi, 2015).

Cette question de l'imaginaire identitaire - de la "communauté imaginée», pour reprendre les termes de B. Anderson- est abordée dans ce dossier par Alice Bombardier qui explore la construction d'un imaginaire national et territorial à partir d'un thème très peu étudié et pourtant central pour comprendre comment les savoirs géographiques construisent, dès l'école, le sentiment national. En comparant divers manuels scolaires parus entre 1973 et 1992, elle interroge les représentations de l'espace géographique, en particulier la carte, surreprésentée dans l'iconographie scolaire. Elle montre que ces cartes sont révélatrices des politiques de construction d'un imaginaire national ancré sur le territoire de l'État-nation, d'abord propre à l'idéologie impériale, puis conforme aux impératifs de la révolution culturelle et au patriotisme, autour de thèmes comme les minorités et le multilinguisme, les rapports villes-campagnes, le changement de point de vue sur la capitale Téhéran de même que sur le reste du monde. Le caractère "insulaire » et "autarcique» de ces cartes, fortement arrimé au patrimoine régional et aux stéréotypes, dénote tout autant une opposition affirmée au passé monarchique et à l'hégémonie occidentale qu'une vision non occidentale du monde. 


\section{Entre réforme de l'État et néolibéralisation : des approches centrées sur les pratiques}

13 Les travaux empiriques récents sont cependant largement urbains et, parmi eux, très majoritairement situés dans la capitale, Téhéran. Parmi eux, on pourra citer les recherches qui, à la croisée de l'anthropologie urbaine, de l'architecture, de l'urbanisme et des visual methodologies, ont renouvelé des thèmes comme les transports, la gouvernance urbaine ou la participation dans les quartiers populaires (Saïdi-Sharouz et Habibi, 2010; Saïdi-Sharouz, 2013). En particulier, plusieurs recherches ont été conduites sur les pratiques de l'espace public du point de vue des femmes, révélant des rapports inédits entre féminismes et espaces publics (Adelkhah, 2000 ; Kian-Thiébaut, 2002 et 2010 ; Amir-Ebrahimi, 2009 ; Saïdi-Sharouz et Guérin-Pace, 2011 ; Direnberger, 2011).

14 Nombre de recherches continuent à construire des terrains sur les pratiques spatiales des Iraniens et des Iraniennes comme les pratiques de mobilité ou les pratiques socioculturelles. Ces entrées par les pratiques révèlent de multiples formes d'adaptation et d'inventivité face aux contraintes et aux opportunités selon les contextes et les groupes sociaux, ce que J.-F. Bayart (2010) nommait «l'autonomie du social ». Le croisement des dimensions sociologique et géographique des pratiques et des politiques culturelles est particulièrement bien représenté (Sreberny et Torfeh, 2016). La géographie des pratiques culturelles est un des nombreux révélateurs des connexions d'échelles entre l'Iran, ses espaces urbains et le reste du monde. Parmi les terrains d'études dans ce domaine, on pourra retenir le théâtre et la littérature (Nanquette, 2017), les arts visuels et le rôle des milieux artistiques d'avant-garde dans les transformations de l'espace (Grigor, 2014; Bombardier, 2012) et, plus encore, le cinéma (Bagheri, 2012 ; Devictor, 2013). Ces études montrent comment s'établissent de nouvelles normes qui jouent comme autant de marqueurs sociaux de l'espace, et interrogent en miroir les espaces de la marginalité et de l'illégalité - on pourra, entre autres, citer un exemple comme celui de la drogue (Ghiabi, 2018).

Parmi ces normes figurent les normes de consommation et d'accès à un style de vie. Le travail proposé dans ce dossier par Mina Saïdi-Sharouz analyse une transformation bien particulière de l'espace urbain de Téhéran: la géographie du commerce (dans le secteur du mobilier et de la décoration) de même que la production d'espaces commerciaux (en centre-ville et en périphérie) et d'espaces résidentiels (programmes de construction de logements neufs). Elle montre que la modification des goûts, valeurs et aspirations des ménages iraniens en matière de logement, d'ameublement et de décoration est inspirée par la diffusion de modèles et de techniques de marketing par le biais des séries télévisées turques, qui apparaissent comme une sorte de compromis entre occidentalisation et tradition, et qui donnent lieu à d'abondantes circulations de personnes et de biens entre les deux pays.

16 Ces approches ont une trame commune qui est, contrairement à ce qu'on pourrait croire, moins une exception iranienne qu'une déclinaison particulière du processus global de néolibéralisation, combinant réformes de l'état (décentralisation, autonomisation des politiques urbaines, réduction des dépenses publiques, brouillage des frontières entre public et privé), dilatation des intérêts et de la rente dans la soustraitance et dans les projets locaux de développement, place croissante de la 
consommation et de la sécurité dans la gestion de l'espace. En d'autres termes, l'économie politique contemporaine trouve en Iran une déclinaison fort intéressante (Harris, 2013 ; Khatam, 2015 ; Khatam et Keshavarzian, 2016 ; Vahabi et Coville, 2017).

17 La recherche de Ronan des Vallières dans ce dossier en est un cas d'étude emblématique. Il s'interroge sur un paradoxe: la prolifération des malls et centres commerciaux à Téhéran et la non viabilité de la grande majorité d'entre eux. Ces espaces commerciaux sont déconnectés de l'activité commerciale ou entrepreneuriale proprement dite mais sont essentiellement des actifs financiers au sein d'un marché immobilier hautement spéculatif et qui n'a cessé de l'être depuis plusieurs décennies. L'intrication des stratégies des acteurs privés (promoteurs et investisseurs) et publics (ministères, municipalité et aménageurs) est tellement étroite, notamment en ce qui concerne les dérogations et la fiscalité, qu'on peut évoquer un «continuum » entre secteurs para-étatique et para-privé, résultat des politiques de pseudo-privatisation. Il s'agit donc d'une illustration particulièrement parlante de financiarisation du foncier ou d'urbanisation du capital.

\section{Pauvreté et vulnérabilité : les mobilités résidentielles dans les rapports ville-campagne}

18 Investiguer les contours de la classe moyenne à partir d'un pays où elle est essentiellement postrévolutionnaire, c'est apporter un éclairage nouveau sur la notion même, dans la mesure où l'après-révolution s'est accompagné d'une démocratisation massive de l'accès à l'éducation et à l'enseignement supérieur. Il y a peu de cas au monde d'une situation postrévolutionnaire aussi stable, qui a de fait permis l'essor d'une nouvelle classe moyenne nombreuse. Pour autant, la situation postrévolutionnaire iranienne est complexe à résumer. D'abord parce que, même si la pauvreté et l'analphabétisme ont considérablement reculé depuis les années 1970, ils existent toujours et, à ce titre, les espaces ruraux, les provinces de confins et les quartiers pauvres sont là pour nous le rappeler. D'autant plus fortement que la paupérisation est incontestable et préoccupante en ce début de millénaire, et qu'elle concerne évidemment les franges vulnérables de la classe moyenne, mais bien plus encore les pauvres.

Dans ce contexte de tension foncière urbaine, investiguer les classes populaires, à partir des quartiers pauvres, du parc de logement social et de la manière dont ils sont pris en compte dans les politiques publiques, est également particulièrement intéressant dans le cas iranien, dans la mesure où les néocitadins pauvres installés dans des conditions précaires, dans les quartiers sud et à la périphérie de Téhéran, ont constitué un groupe social dont la mobilisation a eu un poids politique essentiel au moment de la révolution (Hourcade et Khosrokavar, 1983; Hourcade, 1997 ; SaïdiSharouz, 2013 ; Parsapajouh, 2016). Dans les mentalités, l'Iran des villes et l'Iran des villages sont investis de systèmes de valeurs territoriales différenciés, que l'exode rural et l'extension urbaine ont brouillés.

Dans ce dossier, Amir Amiri présente un cas d'étude qui confirme et renouvelle ces interactions entre villes et campagnes en Iran dans un contexte de pauvreté. L'exode rural vers les grandes villes, en particulier Téhéran, a certes connecté les campagnes avec les ressources urbaines, mais il a aussi privé les villages de main-d'œuvre. En 
parallèle, il montre que l'immigration - il ne faut pas oublier que l'Iran est aussi un pays d'immigration, en majorité afghane - ne concerne pas exclusivement les grandes villes. À partir de terrains villageois situés dans le nord-ouest du pays, il montre que les Afghans ont trouvé à s'employer dans les communes rurales et qu'ils participent à une revitalisation des campagnes et à une complémentarité entre migrations internes et migrations internationales.

21 La paupérisation des ménages les plus vulnérables est de façon patente liée au renforcement des sanctions économiques depuis le milieu des années 2000. Elle est également liée à un recul assumé des dépenses publiques et à une néolibéralisation bien particulière. En effet, comme dans beaucoup d'autres pays, la politique économique intérieure a glissé vers des mesures de flexibilisation, de sous-traitance et de délégation de service public, plus généralement vers un retrait des dépenses de l'État social et une baisse des prestations sociales. Même si c'est sous une déclinaison particulière, on peut y voir une néolibéralisation de l'État, que certains auteurs ont résumé sous l'expression de «l'État sous-traitant» (subcontractor state, pour reprendre les termes de Harris, 2013). Ces cadres expliquent également l'inflexion des politiques urbaines et des grands projets d'aménagement ou de construction qui, comme dans d'autres secteurs, articulent le secteur public et le secteur privé (Khatam, 2015) dans un équilibre spécifique, emblématique de ce que J.-F. Bayart (2010) a décrit comme une «situation thermidorienne", avec une forme de capitalisme privé, de capitalisme d'État, de "ressources administratives", accompagnée d'une intersection des élites économiques, administratives et militaires tout à fait particulière.

Ces éléments de contexte expliquent pourquoi des notions travaillées ailleurs, qui ont considérablement renouvelé les champs thématiques habituels en sciences sociales, trouvent un éclairage nouveau depuis l'Iran, comme la gentrification, les activistes urbains, les connexions entre recherche et pratiques artistiques, etc. Les enjeux politiques continuent d'être significativement corrélés aux questions posées par la fabrique urbaine.

\section{BIBLIOGRAPHIE}

Adelkhah F., 2000. La révolution sous le voile. Femmes islamiques d'Iran. Paris, Karthala, 280 p.

Adelkhah F., 2001. Les Iraniens de Californie : si la République islamique n'existait pas... Paris, SciencesPo, CERI, Les Études du CERI, 75, 40 p.

Adelkhah F., 2012. Les mille et une frontières de l'Iran, quand les voyages forment la nation. Paris, Karthala, 492 p.

Adelkhah F., 2013. Les paradoxes de l'Iran. Idées reçues sur la République islamique. Paris, Le Cavalier bleu, $237 \mathrm{p}$.

Amanat A., Vejdani F. (ed.), 2012. Iran Facing Others: Identity Boundaries in a Historical Perspective. New York, Palgrave Macmillan, 292 p. 
Amir-Ebrahimi M., 2009. Mme Gohari, la dame des quartiers sud de Téhéran. La pensée de midi, 27, p. 33-40.

Bagheri A., 2012. Les relations homme/femme dans le cinéma iranien postrévolutionnaire, stratégies des réalisateurs, analyse sémiologique. Thèse de doctorat en linguistique et sémiologie, Université René Descartes - Paris V, 376 p.

Bayart, J.-F., 2010. La République islamique d'Iran dans la tourmente. Études, tome $413, \mathrm{n}^{\circ} 7$, P. 7-17.

Bombardier A., 2012. Peinture de guerre et représentations anthropomorphiques dans un lieu de prière musulman en Iran. Asiatische Studien, 66-3, p. 565-598.

Bromberger C., 1998. Le football en Iran. Sociétés \& Représentations, 7, p. 101-115.

Chehabi, H. Jafari P., Jefroudi M. (dir.), 2015. Iran in the Middle East. Transnational Encouters and Social History. Londres, Tauris, $336 \mathrm{p}$.

Colby A., Friedlander J. Kelley R., 1993. Irangeles. Iranians in Los Angeles. Oakland, University of California Press, 396 p.

Coville T., 2000. Ordre monétaire et désordres économiques en Iran depuis la révolution islamique. Thèse de doctorat en sciences économiques, Nanterre, Université Paris X, 224 p.

Coville T., 2007. Iran, la révolution invisible. Paris, La Découverte, 264 p.

Devictor A., 2013. Politique du cinéma iranien De l'âyatollâh Khomeyni au président Khâtami. Paris, CNRS Éditions, $310 \mathrm{p}$.

Digard, J.-F. (ed.), 1988, Le fait ethnique en Iran et en Afghanistan. Paris, CNRS, 301 p.

Digard, J.-F., 1981, Techniques des nomades Baxtyâri d'Iran. Paris-Cambridge, Maison des sciences de l'Homme et Cambridge University Press, 273 p.

Digard, J.-F., Hourcade B., Richard Y., 2007 [1996]. L'Iran au XX siècle. Paris, Fayard, 498 p.

Direnberger L., 2011. De la rue à Internet : espaces de contestation féminins et féministes à Téhéran. Justice spatiale Spatial justice, 3 [en ligne]. http://www.jssj.org

Djalili M.-R., 2005. Géopolitique de l'Iran. Bruxelles, Complexe, 144 p.

Djalili M.-R., Kellner T., 2016. Iran : la nouvelle puissance régionale ? Iran le retour. Questions internationales, 77, p. 4-21.

Ghiabi M., 2018. Drogues illégales et gestion de l'espace dans l'Iran moderne. Hérodote, n 169, p. 133-151. DOI:10.3917/her.169.0133

Giblin B. (dir.), 2018. Regards géopolitiques sur l'Iran. Hérodote, 169. 151 p.

Grigor T., 2014. Contemporary Iranian Art: From the Street to the Studio. Londres, Reaktion Books, $256 \mathrm{p}$.

Habibi M., Hourcade B., 2005. Atlas de Téhéran métropole/Atlas of Tehran Metropolis/Atlas-E Kalânshahr-E Tehrân. Vol. 1. La terre et les hommes/Land and people/Sarzamin va mardom. Téhéran, Centre d'informations géographiques de Téhéran (TGIC), 218 p. (en persan), 79 p. (en français), 75 p. (en anglais), 214 cartes.

Harris K., 2013. The Rise of the Subcontractor State: Politics of Pseudo-Privatization in the Islamic Republic of Iran. International Journal of Middle East Studies, vol. 45, n 1, p. 45-70. DOI : $10.1017 /$ S0020743812001250 
Harris K., 2017. A Social Revolution : Politics and the Welfare State in Iran. Oakland, University of California Press, 336 p.

ourcade B., 1997. L'émergence des banlieues de Téhéran. Cahiers d'études sur la Méditerranée orientale et le monde turco-iranien [en ligne], $\mathrm{n}^{\circ} 24$. http://journals.openedition.org/cemoti/1472

Hourcade B. (ed.), 2003. Iran, questions et connaissances. Vol. III. Cultures et sociétés contemporaines. Louvain-Paris, Peeters et Association pour l'avancement des études iraniennes, 318 p.

Hourcade B. et al., 1998. Atlas d'Iran. Montpellier-Paris, Reclus et La Documentation française, $192 \mathrm{p}$.

Hourcade B., Khosrokhavar K., 1983. L'habitat révolutionnaire à Téhéran, 1977-1981. Hérodote, 31, p. 62-83.

Hourcade B., Richard Y., 1993. Les études iraniennes en France. Téhéran, Institut français de recherche en Iran, 90 p. (en français), 58 p. (en persan).

Kashani-Sabet F., 1999. Frontier Fictions: Shaping the Iranian Nation, 1804-1946. Princeton, Princeton University Press, 304 p.

Keddie N. R., Matthee R. P. (ed.), 2002. Iran and the Surrounding World: Interactions in Culture and Cultural Politics. Seattle, University of Washington Press, 408 p.

Khatam A., 2015. Tehran Urban Reforms between Two Revolutions, Developmentalism, Worlding Urbanism and Neoliberalism. Thèse de doctorat, York, Faculty of Environmental Studies, York University, 197 p.

Khatam A., Keshavarzian A., 2016. Decentralization and Ambiguities of Local Politics in Tehran. Middle East Institute, Governing Megacities in MENA and Asia. http://www.mei.edu/content/map/ governing-megacities-mena-and-asia

Kian-Thiébaut A., 2002. Les femmes iraniennes entre Islam, État et famille. Paris, Maisonneuve \& Larose, $318 \mathrm{p}$.

Kian-Thiébaut A., 2010. Le féminisme islamique en Iran : nouvelle forme d'assujettissement ou émergence de sujets agissants? Critique internationale, 46, p. 45-66.

Kiyâni Haftlang K., 2003. The Book of Iran: A Survey on the Geography of Iran. Téhéran, Center for International Cultural Studies, $107 \mathrm{p}$.

Malek A., 2015. Claiming Space: Documenting Second-Generation Iranian Americans in Los Angeles. Anthropology of the Middle East, vol. 13 (2), p. 16-45.

Mervin S., 2007. Les mondes chiites et l'Iran. Paris-Beyrouth, Karthala et IFPO, 484 p.

Moghadam A., 2013. Un espace social transnational entre les Émirats et le sud de l'Iran : les Khodmouni (Lârestâni) à Dubaï. EchoGéo, 25 [en ligne]. http://echogeo.revues.org/13554 DOI : 10.4000/echogeo.13554

Moghadam A., 2016. Tensions diplomatiques irano-arabes et pérennité de la présence des Iraniens à Dubaï. Confluences Méditerranée, 97, p. 63-77.

Moghadam A., Weber S., 2015. Les Iraniens à l'étranger, une composante de la société iranienne. In Moghadam A. et Weber S. (ed.), Diasporas iraniennes. Hommes et migrations [en ligne], 1312. http://www.hommes-et-migrations.fr/index.php?/numeros/l-iran-une-puissance-diasporique/ 7713-les-iraniens-a-l-etranger-une-composante-de-la-societe-iranienne

Moghaddari S., 2015. Migrations et modernités iraniennes. Les familles transnationales. Paris-Téhéran, Éditions du Cygne et IFRI, $172 \mathrm{p}$. 
Mohabbat-Kar R., 2015. Introduction. In Mohabbat-Kar R. (ed.), 2015. Identity and Exile. The Iranian Diaspora between Solidarity and Difference. Berlin, Heinrich Böll Foundation, 148 p.

Mousavi A., Nasiri-Moghaddam N., 2009. Les hauts et les bas de l'archéologie en Iran. La pensée de midi, $\mathrm{n}^{\circ} 27$, p. 137-143.

Naficy H., 1993. The Making of Exile Cultures, Iranian Television in Los Angeles. Minneapolis, University of Minnesota Press, 283 p.

Nanquette L., 2017. The Global Circulation of an Iranian Bestseller. Interventions, vol. 19, $\mathrm{n}^{\circ} 1$, p. 56-72.

Parsapajouh S., 2016. Au cœur d'un bidonville iranien : de Zurâbâd à Islamabâd. Paris-Téhéran, Karthala et IFRI, $361 \mathrm{p}$.

Richard Y., 1980. Le Shi'isme en Iran. Imam et révolution. Paris, Maisonneuve, 135 p.

Saïdi-Sharouz M. (ed.), 2013. Le Téhéran des quartiers populaires. Transformations urbaines et société civile en République islamique. Paris-Téhéran, Karthala et IFRI, 272 p.

Saïdi-Sharouz M., Guerin-Pace F., 2011. La mobilité quotidienne des femmes dans la ville de Téhéran : entre visibilité et invisibilité. L'Espace géographique, tome 40, n² 2, p. 176-188. DOI : 10.3917/eg.402.0176

Saïdi-Sharouz M., Habibi M., 2010. Banlieues sud de Téhéran : L'autoproduction de lieux publics. Urbanisme, 374, p. 35-38.

Salehi-Isfahani D., 2017. Poverty and Income Inequality in the Islamic Republic of Iran. Revue internationale des études du développement, 229, p. 113-136. https://doi.org/10.3917/ried.229.0113

Sreberny A., Torfeh M. (ed.), 2016. Cultural Revolution in Iran: Contemporary Popular Culture in the Islamic Republic. Londres, New York, Tauris, 288 p.

Stone L. (ed.), 2014. Iranian Identity and Cosmopolitanism: Spheres of Belonging. Londres New York, Bloomsbury, $256 \mathrm{p}$.

Vahabi M., Coville T. (dir.), 2017. L'économie politique de la république islamique d'Iran. Revue internationale des études du développement, 229, $182 \mathrm{p}$.

\section{NOTES}

1. Voir, entre autres, le dossier thématique Moyen-Orient: le nouveau "Grand jeu ", Politique étrangère, vol. $81, \mathrm{n}^{\circ} 2$, Paris, Institut français des relations internationales.

\section{INDEX}

Thèmes : Sur le Champ - Sur le Terrain 


\section{AUTEURS}

\section{AMIN MOGHADAM}

Amin Moghadam est géographe au Centre d'études sur le golfe Persique et l'Iran à l'Université de Princeton aux États-Unis. Il a récemment publié :

- Moghadam A., Brones S. (dir.), 2016. Marchés et nouveaux territoires de l'art dans les villes du Sud, Géographie et Cultures, $n^{\circ} 97$ [En ligne]. https://journals.openedition.org/gc/4248

- Moghadam A., Weber S., 2017. Circulating by default. Yerevan and Erbil, the backyards of Iranian mobility. In Vignal L. (dir.), Transnational Middle East. Places, People, Frontiers. Londres, Routledge.

- Moghadam A., 2016. Tensions diplomatiques irano-arabes et pérennité de la présence des Iraniens à Dubaï. Confluences Méditerranée, 2016/2, N 97, p. 63-77.

\section{MINA SAIIDDI-SHAROUZ}

Mina Saïdi-Sharouz, minasaidi@yahoo.com, est architecte et géographe. Enseignante à l'École Nationale Supérieure d'Architecture de Paris-La Villette (ENSAPLV), elle est membre du Laboratoire Architecture Anthropologie (LAVUE/CNRS) et de l'Association Relief. Elle a publié récemment :

- Saïdi-Sharouz M., 2018. L'intégration des migrants dans le centre de Téhéran. La communauté des Ramshehi à Cyrus. In Raulin A., Parsapajouh S. et Blanc-Chaléard M.C. (dir.), Ces villes-là. Actualité de Colette Pétonnet. Paris, Presses Universitaires de Paris Nanterre, p. 135-158.

- Saïdi-Sharouz M., 2015. Les touristes iraniens à Istanbul, les fourmis voyageuses de la mondialisation. Hommes \& Migrations, 2015/4, $\mathrm{n}^{\circ} 1312$, p. 81-89.

- Saïdi-Sharouz M. (dir.), 2013. Le Téhéran des quartiers populaires. Transformation urbaine et société civile en République Islamique. Paris, Karthala-IFRI, 265 p.

\section{SERGE WEBER}

Serge Weber, serge.weber@u-pem.fr, Professeur des universités, Université Paris-Est Marne-laVallée et membre du Laboratoire Analyse comparée des pouvoirs. Il a publié récemment :

- Moghadam A., Weber S., 2016. Circulating by default: Erbil and Erevan, the Backyards of Iranian Mobility. In Vignal L. (dir.), Transnational Middle East: Places, Peoples and Borders. Londres, Routledge, p. 164-180.

- Moghadam A., Weber S. (dir.), 2016. Diasporas iraniennes. Hommes et migrations, $\mathrm{n}^{\circ} 1312$. - Hancock C., Lelévrier C., Ripoll F., Weber S. (dir.), 2016. Discriminations territoriales. Paris, L'œil d'or, $272 \mathrm{p}$. 